\title{
Orientation des traumatisés du membre supérieur (épaule exclue) dès l'accueil en radiologie par l'infirmière organisatrice : étude Ontario
}

\author{
Usefulness of X-ray order for upper limb (shoulder excluded) trauma by the triage nurses
}

\author{
A. Mintandjian - B.I. Mewasing - M. Valentian - E. Burggraff $\cdot$ D. Passot $\cdot$ P. Ray \\ Reçu le 8 octobre 2017; accepté le 6 décembre 2017 \\ (C) SFMU et Lavoisier SAS 2018
}

Résumé Introduction : L'objectif principal de cette étude est de déterminer l'effet d'un protocole de demande anticipée de radiographies par l'infirmier organisateur de l'accueil (IOA) sur le temps de passage des patients au service d'accueil des urgences (SAU).

Patients et méthodes: Cette étude monocentrique prospective a été réalisée en deux phases : une phase-avant (de décembre 2016 à janvier 2017) et une phase-après (de mai à juin 2017), où les patients bénéficiaient d'une demande anticipée de radiographie dès l'IOA. Les patients de plus de 18 ans, valides, ayant consulté au SAU pour un traumatisme du membre supérieur (épaule exclue) de moins de 48 heures et présentant au moins deux critères parmi douleur, tuméfaction, déformation ou impotence fonctionnelle étaient inclus.

Résultats : Deux cent treize patients ont répondu aux critères d'inclusion : 112 en phase 1 et 101 en phase 2. Le temps médian de passage global a diminué de manière significative entre la phase 1 et la phase $2(188$ vs $124 \min , p<0,001)$ ainsi que le temps médian de prise en charge médicale (76 vs 17 min). Plus de 90 \% des infirmiers diplômés d'État (IDE) et plus de $75 \%$ des médecins estiment que le protocole a un intérêt pour diminuer le temps de passage. Un tiers des IDE considère que ce protocole entraîne une surcharge de travail pour l'IOA et une surprescription de radiographies.

Conclusion : Cette étude suggère que l'application d'un protocole de demande anticipée de radiographies par l'IOA

\footnotetext{
A. Mintandjian · B.I. Mewasing · M. Valentian · E. Burggraff ·

D. Passot · P. Ray $(\bowtie)$

Service d'accueil des urgences, hôpital Tenon,

Assistance Publique-Hôpitaux de Paris

55, rue Pelleport, F-75020 Paris, France

e-mail : patrick.ray@tnn.aphp.fr

P. Ray

DHU FAST (Fight Against STress),

Sorbonne université, faculté de médecine

DHU FAST (Fight Against Stress),

F-75013 Paris, France
}

chez les patients ayant un traumatisme du membre supérieur (épaule exclue) permet de réduire le temps de passage au SAU.

Mots clés Membre supérieur - Infirmière organisatrice de l'accueil - Demande anticipée - Radiographie - Urgences . Traumatisme

\begin{abstract}
Introduction: The main objective of this study was to determine the effect of triage nurse initiated X-ray in upper limb trauma on length of stay. Patients and methods: This monocentric, observational, and prospective study was performed in two phases: phase 1-before (from December 2016 to January 2017) and phase 2-after implementation (from May to June 2017), whereas X-ray were ordered by triage nurses. Patients over 18 years of age who had consulted the emergency department (ED) for an upper limb trauma (shoulder excluded) were included.

Results: 213 patients met the inclusion criteria: 112 in phase 1 and 101 in phase 2. Median length of stay has decreased between phase 1 and phase 2 (188 vs $124 \mathrm{~min}, p<0.001$ ). The time delay for medical care has also decreased to $60 \mathrm{~min}$ $(p<0.001)$. More than $90 \%$ of nurses and more than $75 \%$ of medical staff believe that the protocol had a real impact on ED length of stay. One third of the nurses considered that this protocol led to extra work and X-ray over-prescription. Conclusion: This study suggests that nurse initiated X-ray protocol in upper limb trauma (shoulder excluded) leads to a significant decrease in length of stay.
\end{abstract}

Keywords Upper limb $\cdot$ X-ray $\cdot$ Triage nurse $\cdot$ Emergency department · Trauma

\section{Introduction}

Les traumatismes du membre supérieur constituent un motif de consultation fréquent en traumatologie courante [1-4]. Ils 
représentent $32 \%$ des traumatismes selon l'enquête permanente sur les accidents de la vie courante (EPAC) réalisée en 2013 par l'Institut national de veille sanitaire (InVS) auprès de dix services d'accueil des urgences (SAU), dont $53 \%$ de fractures, $32 \%$ de plaies et $25 \%$ de contusions [5]. Près de $80 \%$ des traumatisés du membre supérieur retournent au domicile. On retrouve très peu de données sur l'épidémiologie précise des traumatismes du membre supérieur. Selon une étude épidémiologique nord-américaine, les traumatismes des différents segments du membre supérieur sont, par ordre de fréquence, les traumatismes des doigts (38\%) suivis par l'épaule (17\%), le poignet (15\%) et le coude. Le traumatisme de membre implique fréquemment une radiographie [6]. Habituellement, ce sont le mécanisme lésionnel et l'examen clinique qui dictent la décision du médecin de réaliser ou non une radiographie. La demande anticipée de radiographies par l'infirmier organisateur de l'accueil (IOA), principalement en traumatologie, est une pratique qui a déjà fait l'objet de diverses évaluations internationales [7-10], surtout dans l'entorse de cheville encadrée par les critères d'Ottawa [11-12]. Néanmoins, les études françaises d'évaluation sont rares. En 2016, dans notre SAU, nous avons rapporté, lors d'une étude monocentrique avant/après, l'intérêt de la mise en place d'un protocole de demande anticipée de radiographies dans les entorses de cheville en utilisant les critères d'Ottawa, avec une diminution de près de $50 \%$ du temps de passage global [12]. Cependant, il n'existe pas, à notre connaissance, de protocole standardisé regroupant des critères précis et validés pour la prescription de radiographies chez les traumatisés du membre supérieur.

Le but de cette étude était d'évaluer l'intérêt de la mise en place d'un protocole de demande anticipée de radiographies par l'IOA chez les patients qui consultent pour un traumatisme isolé du membre supérieur (épaule exclue). Le critère de jugement principal de l'étude était la diminution du temps de prise en charge médicale et du temps de passage global de ces patients après mise en place du protocole.

\section{Patients et méthodes}

Ce travail de type avant/après est une étude monocentrique, prospective, observationnelle, réalisée au sein du CHU de Tenon qui a accueilli environ 44000 patients adultes en 2016. Sur le plan éthique, cette étude entrait dans le cadre de la loi Jardé type 3 (décret $n^{\circ}$ 2016-1537 du 16 novembre 2016) et a fait l'objet d'une demande auprès du Comité de protection des personnes (CPP) Île-de-France $\mathrm{n}^{\circ} 2$ le 31 mars 2017 pour la phase prospective. Elle a été évaluée par le bureau «Qualité des pratiques et recherche biomédicale » qui a conclu que le protocole relevait d'une évaluation de pratiques professionnelles et non d'un projet visant à développer les connaissances biologiques ou médicales au sens de la loi relative aux recherches impliquant la personne humaine (RIPH) ; il ne relevait donc pas de l'avis d'un comité de protection des personnes (CPP). Les patients étaient informés par l'IOA sur le protocole et le caractère dérogatoire de la demande anticipée de radiographies. Seul le consentement oral du patient a été recueilli.

Ce travail a été réalisé en deux phases sur une période de deux mois respectivement, choisie de manière arbitraire : phase 1 rétrospective (avant mise en place du protocole) du $1^{\text {er }}$ décembre 2016 au 31 janvier 2017 et phase 2 prospective (après mise en place du protocole) du $1^{\mathrm{er}}$ mai au 30 juin 2017. Entre $10 \mathrm{~h}$ et $20 \mathrm{~h}$, les jours de semaine, les patients sont habituellement pris en charge par le médecin d'accueil et d'orientation (MAO) qui gère le « circuit court » : traumatologie et consultation de médecine générale (tri 4 et 5 de la classification infirmière des malades aux urgences (CIMU), donc patients valides et non hyperalgiques). Pour des raisons de faisabilité, nous nous sommes limités aux traumatismes isolés du membre supérieur relatifs au coude, au poignet et à la main. Nous avons exclu les traumatismes de l'épaule, car l'examen clinique de l'épaule est complexe et ne semblait pas être approprié à une évaluation à l'accueil des urgences par l'IOA. Tout patient de plus de 18 ans, valide, stable sur le plan clinique, ayant consulté au SAU pour un traumatisme du membre supérieur (épaule exclue) datant de moins de 48 heures et présentant au moins deux des critères suivants : douleur, tuméfaction (œdème, ecchymose, hématome), déformation ou impotence fonctionnelle, était inclus dans l'étude Ontario (orientation des traumatisés du membre supérieur [épaule exclue] dès l'accueil en radiologie par l'infirmier organisateur). Étaient exclus de l'étude les patients agités (sous l'emprise de toxiques et/ou d'alcool, agressifs, hyperalgiques), les détenus, les mineurs, les femmes enceintes, les patients hyperalgiques $(\mathrm{EN}>8)$ ainsi que les patients nécessitant une hospitalisation, se présentant pour un traumatisme résultant d'une cinétique élevée, un polytraumatisme, un traumatisme datant de plus de 48 heures, ou les patients allongés sur un brancard dès l'IOA.

Cette délégation de compétences entre professionnels de santé a reçu l'aval et la validation de notre direction des soins infirmiers ainsi que celle du service de radiologie. Tous les IDE formés au poste d'IOA ont reçu une formation de trente minutes sur l'anatomie du membre supérieur (hormis l'épaule) et les indications de demande anticipée de radiographies en fonction des critères définis par le groupe de travail (Annexe A). Cette formation a été réalisée entre les deux phases de l'étude par deux médecins du service, expérimentés et diplômés du DIU d'urgences traumatologiques. Plusieurs séances ont été proposées pour former le maximum d'IDE. La formation a d'abord été testée auprès d'un échantillon composé d'une cadre infirmière, des trois infirmiers référents du protocole ainsi que de deux médecins. Elle a ensuite été soumise à un questionnaire auprès des 
IDE formées afin d'en améliorer le contenu. Le bon de demande anticipée, élaboré par l'équipe référente, représente un schéma du membre supérieur (Fig. 1). Ce protocole s'est inscrit dans une procédure IOA plus large et standardisée, associant antalgie, glaçage et immobilisation du membre supérieur, dont l'objectif était une amélioration des soins.
Sur le schéma (Fig. 1), l'IOA détaille alors l'existence d'une douleur/tuméfaction/déformation/impotence fonctionnelle en regard de l'articulation ou du membre concerné en faisant une croix. Selon les critères présents, elle demande une radiographie face et profil de l'articulation ou du membre concerné (coude, poignet et/ou main et doigts) en
IDENTIFICATION DU PATIENT

Etiquette à coller
SERVICE D'ACCUEIL DES URGENCES

Pr Ray

\section{DEMANDE D'EXAMEN RADIOLOGIQUE URGENT}

Date : Heure : Signature :
Grossesse en cours : oui / non

1/ Question posée : recherche de fracture

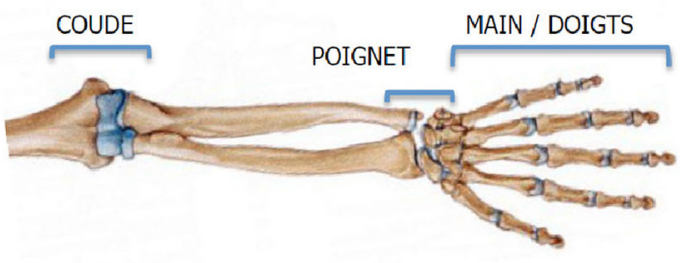

2/ Urgence :

\begin{tabular}{|c|c|c|}
\hline COUDE & POIGNET & MAIN / DOIGTS \\
\hline$\square$ Douleur & $\square$ Douleur & $\square$ Douleur \\
\hline$\square$ Déformation & $\square$ Déformation & $\square$ Déformation \\
\hline$\square$ Tuméfaction & $\square$ Tuméfaction & $\square$ Tuméfaction \\
\hline $\begin{array}{l}\square \text { Impotence } \\
\text { fonctionnelle }\end{array}$ & $\begin{array}{l}\square \text { Impotence } \\
\text { fonctionnelle }\end{array}$ & $\begin{array}{l}\square \text { Impotence } \\
\text { fonctionnelle }\end{array}$ \\
\hline $\begin{array}{l}\square \text { Flexion extension } \\
\square \text { Prono supination }\end{array}$ & $\square$ Flexion extension & $\begin{array}{l}\square \text { Flexion } \\
\text { extension }\end{array}$ \\
\hline
\end{tabular}

\begin{tabular}{|c|c|c|}
\hline$\square$ Coude Face + Profil & $\square$ Poignet Face + Profil & $\square$ Main $F+P$ \\
\hline $\begin{array}{l}\square \mathrm{D} \\
\square \mathrm{G}\end{array}$ & $\begin{array}{l}\square \mathrm{D} \\
\square \mathrm{G}\end{array}$ & $\begin{array}{l}\square \mathrm{D} \\
\square \mathrm{G}\end{array}$ \\
\hline & & $\begin{array}{c}\square \text { Doigts } F+P \\
\square \mathrm{D} \\
\square \mathrm{G}\end{array}$ \\
\hline
\end{tabular}

Fig. 1 Demande anticipée pour l'infirmière organisatrice de l'accueil (IOA) 
précisant la latéralité. Ce document était archivé dans une bannette dédiée au sein du service de radiologie puis collecté par les médecins référents du protocole pour les inclusions de la phase 2. Le patient est ensuite orienté vers le service de radiologie afin de faire les radiographies demandées par l'IOA. La prise en charge médicale n'intervient que dans un second temps après le retour du patient du service d'imagerie (Fig. 2). La consultation médicale unique regroupe alors l'interrogatoire, l'examen clinique et l'examen des radiographies effectuées juste après l'accueil par l'IOA.

Le recueil des données s'est effectué par le logiciel Urqual $^{\circledR}$ (Maincare Solutions, Cestat, France). Pour la phase 1, les patients ont été sélectionnés par le motif IOA « traumatisme du membre supérieur ». Pour la phase 2, les patients ont été inclus en récupérant les bons de demande anticipée de radiographies auprès du service de radiologie. Une vérification a été effectuée via Urqual ${ }^{\circledR}$ en utilisant le motif IOA « Protocole Ontario » créé spécifiquement à cet effet. Cela a permis de retrouver un certain nombre de dossiers. Une enquête d'évaluation sur la faisabilité et l'impact du protocole au SAU a été menée par l'équipe référente et envoyée aux IDE et à l'équipe médicale. Elle prenait la forme d'un questionnaire anonyme, élaboré avec l'outil formulaire de Google Drive ${ }^{\circledR}$, avec des questions fermées. Le choix de ce type de support a été motivé par la rapidité de distribution par mail et la simplicité de traitement des données.

\section{Analyse statistique}

En nous appuyant sur les données de la littérature [13] et en faisant l'hypothèse d'une diminution de $36 \%$ du temps de passage, nous avons calculé que le nombre minimal de patients à inclure pour montrer une différence significative avec $p$ inférieur à 0,05 et une puissance de $90 \%$ était de 118 (soit 59 dans chaque groupe). Il a été calculé à l'aide du site BiostaTGV. Nous avons vérifié l'égalité des populations à l'aide du test non paramétrique de Mann-Whitney pour l'âge (variable quantitative) et du test non paramétrique du Chi ${ }^{2}$ pour le sexe (variable qualitative). Nous avons ensuite effectué nos tests statistiques sur les durées (variables quantitatives continues) en utilisant le test paramétrique $t$ de Student. Nous avons étudié les moyennes, les médianes, les écartstypes et les minina/maxima résultant des données continues issues de chaque groupe. Les comparaisons statistiques ont été effectuées en situation bilatérale. Une valeur de $p$ inférieure à 0,05 a été considérée comme statistiquement significative.

\section{Résultats}

En 2016, 3,3\% des patients ont consulté au SAU du CHU de Tenon pour un traumatisme du membre supérieur. Au total, 213 patients ont été inclus : 112 patients en phase 1 et 101 en phase 2 (Fig. 3). Les caractéristiques des patients étaient comparables dans les deux groupes (Tableau 1). Les diagnostics posés dans les deux groupes étaient comparables, principalement des contusions et des fractures. Le temps médian de passage global a diminué de manière significative entre la phase 1 et la phase $2(188$ vs $124 \mathrm{~min}, p<0,001)$ ainsi que le temps médian de prise en charge médicale (76 vs $17 \mathrm{~min}$ ). Une analyse en sous-groupe en fonction de l'heure de consultation des patients (jour ou nuit) a également mis en évidence une diminution significative du temps de prise en

\section{AVANT}

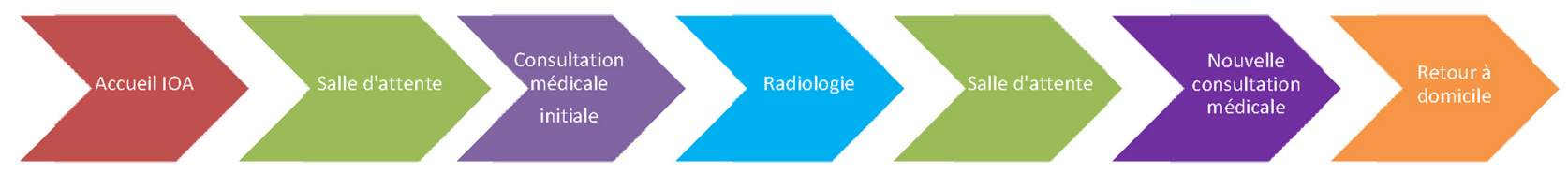

I

\section{APRES}

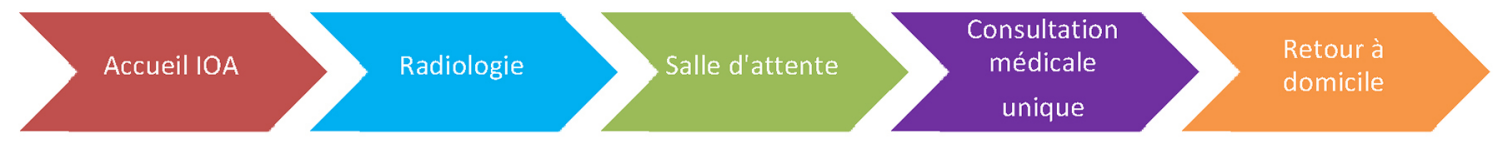

Fig. 2 Circuit du patient avant et après protocole de demande anticipée de radiographies 

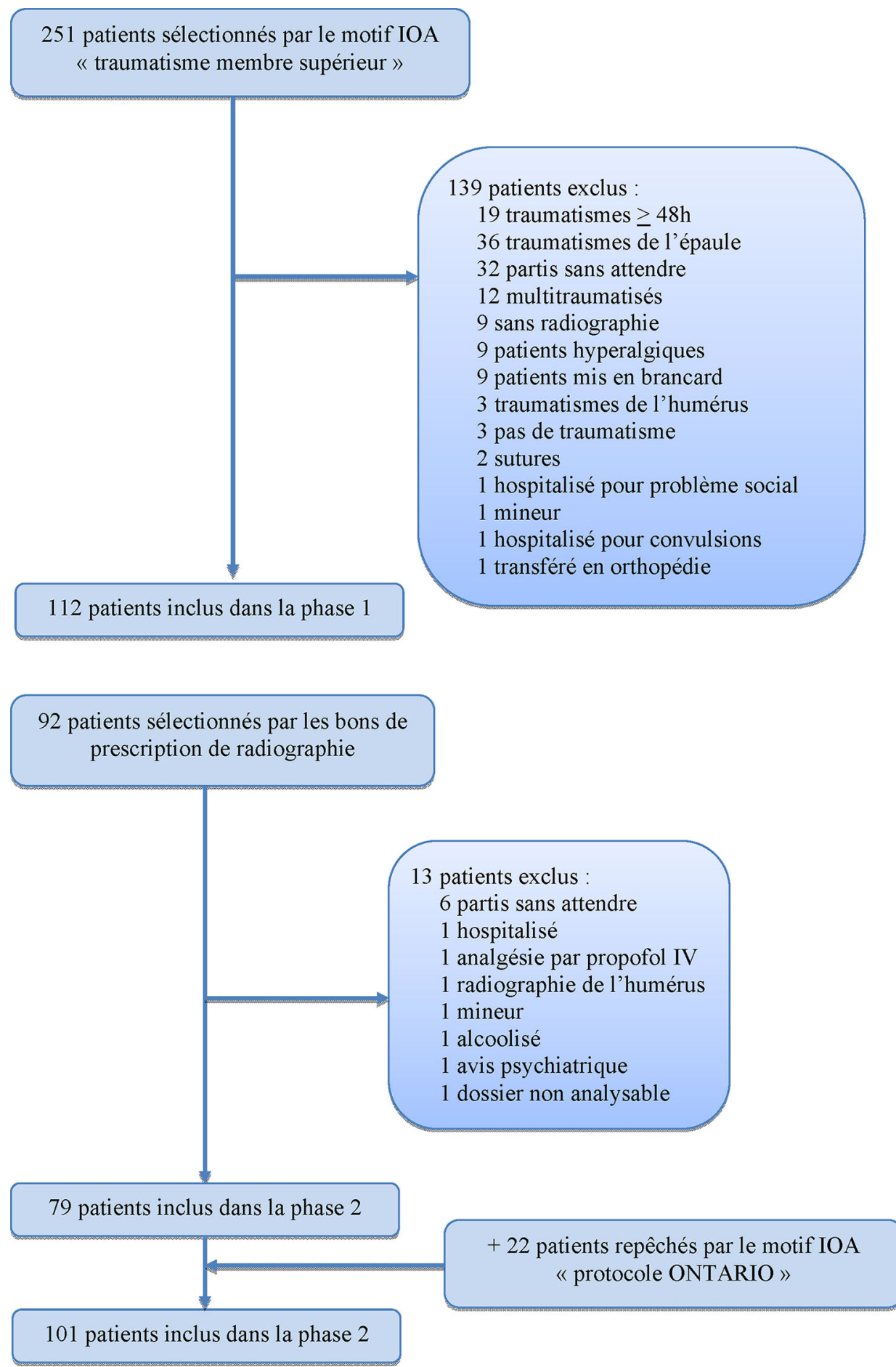

Fig. 3 Diagrammes de flux des phases 1 et 2

charge médicale et du temps de passage global dans les deux sous-groupes (Tableau 2).

Vingt-trois IOA ont reçu la formation Ontario et ont répondu à un premier questionnaire. Treize médecins ( $86 \%)$ ont répondu au questionnaire d'évaluation du protocole (Tableau 3). Les résultats montrent que la formation reçue était suffisante avec des intérêts et objectifs clairs et bien compris par les IDE présents. Au terme de l'étude, les 23 IDE formés au poste IOA ont répondu au questionnaire d'évaluation du protocole (Tableau 3). Une formation continue s'avère nécessaire selon $57 \%$ des IDE. En outre, $78 \%$ des IDE ont interprété ce protocole comme une valorisation de leur métier. Enfin, $91 \%$ des IOA et $100 \%$ des médecins ont été satisfaits des protocoles de radiographies anticipées 
mis en place dans le service. Néanmoins, on observe qu'un tiers des IDE interrogés estime que la demande anticipée de radiographies est une surcharge de travail pour l'IOA. Les raisons invoquées sont : la recherche des critères d'exclusion et d'inclusion, le bon de radiographie à remplir, l'accompagnement du patient en radiologie par l'aide-soignant, de l'accueil ainsi qu'au retour, d'autant plus en cas d'affluence.

\section{Discussion}

Dans ce travail, nous avons montré que le temps moyen de passage global du patient traumatisé du membre supérieur (épaule exclue) diminue de $30 \%$ avec la mise en place d'un protocole de demande anticipée de radiographies par l'IOA. Cette réduction est essentiellement due à une diminution du temps de prise en charge médicale, qui est réduit de moitié. Néanmoins, à la différence de notre précédent travail sur les entorses de cheville, le temps de prise en charge avant le premier contact médical ne varie pas [12]. Cette réduction du temps de passage global s'explique par la modification du circuit de prise en charge du patient au SAU : cinq étapes contre sept étapes (Fig. 1). Sur ce schéma, les étapes avant le premier contact médical passent de « accueil IOA + salle d'attente » à « accueil IOA + radiologie + salle d'attente »,

\begin{tabular}{|c|c|c|c|}
\hline & $\begin{array}{l}\text { Groupe } 1 \\
n=112\end{array}$ & $\begin{array}{l}\text { Groupe } 2 \\
n=101\end{array}$ & Valeur de $p$ \\
\hline Âge (années) & $41 \pm 17$ & $38 \pm 16$ & 0,11 \\
\hline Sexe masculin & $54(48)$ & $62(61)$ & 0,054 \\
\hline Diagnostics & & & 0,49 \\
\hline Fracture & 49 (44) & $43(43)$ & \\
\hline Luxation & $3(3)$ & $6(6)$ & \\
\hline Contusion & $60(53)$ & $52(52)$ & \\
\hline $\begin{array}{l}\text { Heure } \\
\text { de consultation }\end{array}$ & & & 0,11 \\
\hline $10 \mathrm{~h}$ à $20 \mathrm{~h}$ & $81(72)$ & $62(61)$ & \\
\hline $20 \mathrm{~h}$ à $10 \mathrm{~h}$ & $31(28)$ & 38 (39) & \\
\hline
\end{tabular}

avec mise à profit pour effectuer un examen complémentaire (radiographie ici) très probablement demandé. Il en résulte une amélioration du flux de ces patients souffrant d'un traumatisme isolé du membre supérieur.

Nous avons comparé les ressentis des IDE et des médecins, acteurs centraux et complémentaires de ce protocole. Deux résultats ont particulièrement attiré notre attention. Tout d'abord, un tiers des IDE concernés et un tiers des médecins interrogés estiment que ce protocole représente une surcharge de travail pour l'IOA. Les raisons principales sont la recherche des critères d'exclusion et d'inclusion, le remplissage du bon de radiographie et l'accompagnement du patient en radiologie par l'aide-soignant. Le temps alloué à la demande anticipée de radiographies sur le temps de tri global de l'IOA n'a pas été évalué, mais l'équipe de Sorenson et al. avait montré en 2011 qu'elle n'allongeait pas de manière significative le temps de passage à l'IOA des patients au SAU [10]. Les patients inclus dans le protocole Ontario sont des patients « ambulatoires » qui devraient pouvoir se rendre dans le service de radiologie par leurs propres moyens. Une réflexion sur la signalétique entre box IOA/service de radiologie/salle d'attente permettrait de faciliter les déplacements des patients. Ces problématiques montrent que la mise en place de ce protocole impose une modification du parcours du patient et nécessite d'impliquer tous les maillons de la chaîne : l'IOA, le médecin, l'aide-soignant, le manipulateur radio et le patient qui a un rôle central puisqu'il est valide.

Nous pensons que les critères de demande anticipée de radiographies que nous avons définis, fondés sur la littérature, se sont révélés adaptés. En effet, si un tiers des IDE pensent que le protocole entraîne une surprescription de radiographies, aucun des médecins ne le pense. Plusieurs études dans la littérature rapportent une bonne adéquation entre IOA et médecins en ce qui concerne les demandes de radiographies suivant l'application d'un protocole [14-26]. Il est conseillé d'avoir un programme de formation précis et un protocole standardisé : quand les IOA sont formés, les études montrent que $98 \%$ d'entre eux adhèrent à la mise en place d'un tel protocole, et que cela permet une réduction du nombre de radiographies, donc une diminution des coûts, de l'exposition aux radiations des patients, et enfin une diminution du temps de consultation médicale [7,9].

Tableau 2 Résultats principaux

\begin{tabular}{|llll|}
\hline & Groupe $\mathbf{1}$ & Groupe $\mathbf{2}$ & Valeur de $\boldsymbol{p}$ \\
$\boldsymbol{n}=\mathbf{1 1 2}$ & $188(93-265)$ & $124(76-198)$ & $<0,001$ \\
\hline Temps de passage global (min) & $76(47-114)$ & $17(7-49)$ & $<0,001$ \\
Temps de prise en charge médicale (min) & $71(29-145)$ & $80(47-149)$ & 0,42 \\
Temps de PEC avant $\mathbf{1}^{\text {er }}$ contact médical (min) & & \\
\hline Les données sont exprimées en médiane (interquartile). PEC : prise en charge & & \\
\hline
\end{tabular}


Tableau 3 Résultats du questionnaire d'évaluation IDE versus médecins

\begin{tabular}{|lll|}
\hline & $\begin{array}{l}\text { IDE } \\
\boldsymbol{n}=\mathbf{2 3}\end{array}$ & $\begin{array}{l}\text { Médecin } \\
\boldsymbol{n}=\mathbf{1 3}\end{array}$ \\
\hline $\begin{array}{l}\text { Intérêt de la demande anticipée } \\
\text { de radiographies sur : }\end{array}$ & \\
$\quad \begin{array}{l}\text { Diminution du temps d'attente } \\
\quad \text { Diminution du temps de passage }\end{array}$ & $21(91)$ & $10(77)$ \\
$\quad$ global & & $11(85)$ \\
$\quad$ Amélioration de la prise en charge & $21(91)$ & $11(85)$ \\
$\begin{array}{l}\text { La formation initiale a-t-elle été } \\
\text { suffisante } ?\end{array}$ & $18(80)$ & NC \\
$\begin{array}{l}\text { Compréhension de l'intérêt } \\
\text { et les objectifs de ce protocole }\end{array}$ & $22(96)$ & $13(100)$ \\
Inconvénient en termes de : & & \\
$\quad \begin{array}{l}\text { Surcharge de travail pour l'IOA } \\
\quad \text { Surprescription de radiographies }\end{array}$ & $8(35)$ & $5(38)$ \\
$\quad$ Non-adhésion d'un patient & $1(4)$ & $0(0)$ \\
Poursuite de ce type de protocole & $21(91)$ & $13(100)$ \\
\hline $\begin{array}{l}\text { Les données sont exprimées en nombre (\%). IDE : Infirmier } \\
\text { diplomé d'État. IOA : Infirmier organisateur de l'accueil }\end{array}$
\end{tabular}

Nous sommes conscients que cette étude présente plusieurs limites. Tout d'abord une limite juridique : depuis le 31 mars 2012, en France, les infirmiers sont autorisés à prescrire certains dispositifs médicaux dont la liste est fixée par l'arrêté du 20 mars 2012 [27]. Cependant, la prescription de médicaments et d'imagerie ne relève pas de leurs compétences propres et nécessite des protocoles de service. Ainsi, en France, la prescription par délégation du médecin relève de l'article L. 4011-1 du Code de la santé publique [28]. Cet article permet aux " professionnels de santé de s'engager dans une démarche de coopération ayant pour objet d'opérer entre eux des transferts d'activité ou d'actes de soins" : cela permet donc une délégation de tâches entre professionnels médicaux et paramédicaux, raison pour laquelle nous utilisons ici le terme de demande anticipée (et non pas de prescription). Il existe parfois un décalage entre les horaires de « log » (prise en charge médicale et surtout nonadmission) et la réalité. En effet, certains dossiers étaient conclus en «non-admission » avant même que le patient soit revenu du service de radiologie. Ces événements ont pu survenir dans les deux groupes de façon aléatoire. Cependant, certains dossiers ont dû être exclus car, par exemple, le médecin se loguait avant l'IOA et cela faussait toute analyse. L'impact de la mise en place du protocole sur le nombre de radiographies prescrites n'a pas été étudié. En effet, la réalisation d'une radiographie faisait partie des critères d'inclusion dans l'étude. Une réponse partielle à cette question réside dans le questionnaire médecin : aucun des médecins interrogés n'estimait que le protocole entraîne une surprescription de radiographie. Nous n'avons pas fait d'analyse en sous-groupe d'âge ou selon la région anatomique lésée.

\section{Conclusion}

Cette étude suggère que l'application d'un protocole de demande anticipée de radiographies par l'IOA chez les patients ayant un traumatisme du membre supérieur (épaule exclue) permet de réduire le temps de passage global au SAU. Cela implique une formation initiale des IOA qui devra être continue et une sensibilisation de tous les acteurs de la chaîne du SAU, ainsi que du service de radiologie.

Liens d'intérêts : les auteurs déclarent ne pas avoir de liens d'intérêts.

\section{Références}

1. Carrasco V, Baubeau D (2003) Les usagers des urgences. Premiers résultats d'une enquête nationale. DREES, Études et résultats $\mathrm{n}^{\circ}$ 212. http://drees.solidarites-sante.gouv.fr/IMG/pdf/er212. pdf (Dernier accès le 6 octobre 2017)

2. Baubeau D, Carrasco V (2003) Motifs et trajectoires de recours aux urgences hospitalières. DREES, Études et résultats $n^{\circ} 215$. http://www.sfmu.org/upload/referentielsSFMU/er215.pdf (Dernier accès le 6 octobre 2017)

3. Pitts SR, Niska RW, Xu J, Burt CW (2008) National hospital ambulatory medical care survey: 2006 emergency department summary. Natl Health Stat Report 7:1-39

4. Raphaël M (2003) Organisation des urgences traumatiques dans un site d'accueil. Séminaire SFMU. Traumatologie d'urgence : de l'accueil à l'orientation. http://www.sfmu.org/upload/70 formation/02_eformation/03 journees/archives/brochure.pdf (Dernier accès le 6 octobre 2017)

5. Institut national de veille sanitaire (InVS) (2015) Description et incidence des accidents de sport. Enquête permanente sur les accidents de la vie courante 2013. http://invs.santepubliquefrance.fr/Dossiers-thematiques/Maladies-chroniques-et-traumatismes/Traumatismes/Bases-de-donnees-outils/Enquete-Permanentesur-les-Accidents-de-la-Vie-Courante-EPAC (Dernier accès le 6 octobre 2017)

6. Ootes D, Lambers KT, Ring DC (2012) The epidemiology of upper extremity injuries presenting to the emergency department in the United States. Hand (N Y) 7:18-22

7. Cauterman M, Raphael M, Miroux P (2008) Prévalence, motifs et modalités de mise en œuvre d'une prescription anticipée d'examens d'imagerie par les infirmières dans les services des urgences en France. J Eur Urg 25:757-8

8. Lee WW, Filiatrault L, Abu-Laban RB, et al (2016) Effect of triage nurse initiated radiography using the Ottawa ankle rules on emergency department length of stay at a tertiary centre. CJEM 18:90-7

9. Allerston J, Justham D (2000) A case-control study of the transit time through an accident and emergency department of ankle injured patients assessed using the Ottawa Ankle Rules. Accid Emerg Nurs 8:148-54 
10. Sorensen EL, Keeling A, Snyder A, Syverud S (2012) Decreasing ED length of stay with use of the Ottawa Ankle Rules amongst nurses. J Emerg Nurs 38:350-2

11. Stiell IG, Greenberg GH, McKnight RD, et al (1992) A study to develop clinical decision rules for the use of radiography in acute ankle injuries. Ann Emerg Med 21:384-90

12. Valentian M, Mewasing IB, Burggraff E, et al (2017) Usefulness of X-ray order for ankle trauma by the triage nurses. Ann Fr Med Urgence 7:77-84

13. Lindley-Jones M, Finlayson BJ (2000) Triage nurse requested X-rays-the result of a national survey. J Accid Emerg Med $17: 108-10$

14. Tambimuttu J, Hawley R, Marshall A (2002) Nurse-initiated X-ray of isolated limb fractures in the emergency department: research outcomes and future directions. Aust Crit Care 15: $119-22$

15. Ching K, Leng L, See N (1999) Initiation of X-rays by the triage nurse: competency and its effect on patient's total time spent in the A \& E department. Singapore Nurs J 263:22-6

16. Lee KM, Wong TW, Chan R, et al (1996) Accuracy and efficiency of X-ray requests initiated by triage nurses in an accident and emergency department. Accid Emerg Nurs 4:179-81

17. Macleod AJ, Freeland P (1992) Should nurses be allowed to request X-rays in an accident \& emergency department? Arch Emerg Med 9:19-22

18. Ward W (1999) Key issues in nurse requested X-rays. Emerg Nurse 6:19-23

19. Kelly AM, McCarthy S, Richardson S, et al (1995) Triage nurse initiated X-rays for limb injuries are accurate and efficient. Emerg Med Australas 7:81-4
20. Parris W, McCarthy S, Kelly AM, Richardson S (1997) Do triage nurse initiated X-rays for limb injuries reduce patient transit time? Accid Emerg Nurs 5:14-5

21. Patel H, Celenza A, Watters T (2012) Effect of nurse initiated $\mathrm{X}$-rays of the lower limb on patient transit time through the emergency department. Australas Emerg Nurs J 15:229-34

22. Jayaprakash N, O’Sullivan R, Bey T, et al (2009) Crowding and delivery of health care in emergency departments: the European perspective. West J Emerg Med 10:233-9

23. Hunter D (2010) Triage nurse X-ray protocols for hand and wrist injuries. Emerg Nurs 17:20-4

24. Solomone JA, Langford A, Patty W (1997) The application of the Ottawa Ankle Rules by advance practice nurses in an emergency department fast track. Acad Emerg Med 4:409

25. Kec RM, Richman PB, Szucs PA, et al (2003) Can emergency department triage nurses appropriately utilize the Ottawa Knee Rules to order radiographs? An implementation trial. Acad Emerg Med 10:146-50

26. Mann CJ, Grant I, Guly H, Hughes P (1998) Use of the Ottawa ankle rules by nurse practitioners. J Accid Emerg Med 15:315-6

27. République Française (2012) Arrêté du 20 mars 2012 fixant la liste des dispositifs médicaux que les infirmiers sont autorisés à prescrire (en ligne). In Journal officiel de la République française $\mathrm{n}^{\mathrm{o}} 0077$ du 30 mars 2012, page 5768, texte $\mathrm{n}^{\mathrm{o}} 34$. https://www. legifrance.gouv.fr/eli/arrete/2012/3/20/ETSH1208335A/jo (Dernier accès le 13 novembre 2017)

28. République Française (2017) Article L. 4011-1 du Code de la santé publique (en ligne). https://www.legifrance.gouv.fr/affich CodeArticle. do;jsessionid $=620815$ A798BC9CA6DBE6248009 DFE822.tpdila09v_1?idArticle=LEGIARTI000028393846\&cid Texte $=$ LEGITEXT000006072665\&categorieLien=id\&dateTexte (Dernier accès le 13 novembre 2017) 